\title{
Severity of Disease from COVID-19 in Patients with Obesity and MAFLD: Is there an association?
}

\author{
Muhammad Kamran ${ }^{1}$ and Wasim Jafri \\ ${ }^{1}$ Department of Gastroenterology, Fazaia Ruth Pfau Medical College and PAF Hospitals, Karachi, Pakistan \\ ${ }^{2}$ Department of Medicine, The Aga Khan University Hospital, Karachi, Pakistan
}

The coronavirus disease of 2019 (COVID-19) pandemic has so far proved to be the worst health-related calamity seen by mankind since the 20th century Spanish Flu. Apparently, originating in late 2019 from the Chinese province of Wuhan, this viral disease has since then spread globally in a matter of weeks, if not days. ${ }^{1}$ Currently, majority of the world nations are overwhelmed with this catastrophe, not only in terms of its impact on the healthcare system, but also the fiscal devastation that the pandemic has caused, for instance loss of jobs and small businesses etc. At the time of writing this review, total number of confirmed cases infected with COVID-19 have exceeded 12 million, with more than half a million reported deaths because of the disease itself or its complications. The situation in Pakistan is also alarming, with approximately 2,50,000 confirmed cases and nearly 5000 deaths across the country.

The causative organism in case of COVID-19 infection is severe acute respiratory syndrome coronavirus 2 (SARS-CoV-2). The first decade of this millennium witnessed the outbreak of SARSCoV, which also started from China, but ultimately spread in 26 countries across five continents. ${ }^{2}$ SARS-CoV involved mainly the respiratory system; therefore, it was naturally assumed by most authorities that SARS-CoV-2 would also have a similar clinical spectrum, resulting in fever, dyspnea and cough. ${ }^{3}$ However, we now know that SARS-CoV-2 behaves like a systemic illness, and has the potential of involving other vital organs, especially theliver. ${ }^{4}$

Non-alcoholicfatty liverdisease (NAFLD) is globally being recognised as one of the most common causes of chronic liver disease, and is often associated with obesity. ${ }^{5}$ As the lacunae in understanding the pathogenesis of NAFLD are increasingly being filled, a new terminology named "MAFLD" (metabolic dysfunction-associated fatty liver disease) has been coined by experts in the field in a recentconsensus statement. ${ }^{6}$

Correspondence to: Dr. Muhammad Kamran, Department of Gastroenterology, Fazaia Ruth Pfau Medical College and PAF Hospitals, Karachi, Pakistan

E-mail: muhammadkamran81@gmail.com

Received: July 23, 2020; Revised: August 08, 2020;

Accepted: August 08, 2020

DOI: https://doi.org/10.29271/jcpsp.2020.09.891
As with various other viral illnesses, variable degrees of liver dysfunction, mostly in the form of elevated transaminase levels [alanine aminotransferase (ALT) and aspartate aminotransferase (AST)], are commonly found in patients with COVID-19. ${ }^{7}$ The contributing factors to this liver injury include hypoxia (secondary to COVID pneumonia), the systemic inflammatory response which is part of the syndrome (usually cell-mediated), and the use of various drugs as potential anti-viral or supportive treatments in hospitalised patients, which damage the hepatocytes. ${ }^{8}$ There are other possible mechanisms of liver damage being extensively studied, including the role of angiotensin-converting enzyme 2 (ACE2) receptors in bile ductal epithelial injury. ${ }^{9}$ Whether patients with underlying liver cirrhosis are at a higher risk of developing further deterioration in liverfunction is still a matter of debate. ${ }^{7}$ However, pre-pandemic studies have shown that such patients have a higher mortality when they develop acute respiratory distress syndrome (ARDS) as opposed to patients with no underlying liver disease..$^{10}$ In this context, given the high prevalence of obesity and MAFLD, analysing the severity of COVID-19 infection in the presence of underlying fatty liver disease becomes even more crucial, as this can drastically affect the overall disease outcome in such individuals.

Over the years, obesity has become a major population health issue. Previously thought of as a problem of the developed world, many under-developed countriesare also facing thechallenge of obesity and its complications, mainly because of the shift to a more sedentary lifestyle, absence of physical activity, and consumption of high-calorie processed foods. ${ }^{11}$ Obese individuals are at a higher risk of developing respiratory diseases like obstructive sleep apnea, obesity hypoventilation syndrome, pulmonary embolism (resulting from deep venous thrombosis) and even community-acquired pneumonia. ${ }^{12}$ Therefore, much interest has been evinced in finding out an association between obesity and severity of COVID-19 infection.

A study conducted in three hospitals in China during the early days of the pandemic clearly showed that obese subjects had more severe form of illness reflected by high C-reactive protein (CRP) and lymphopenia. These obese COVID-19 positive patients also had a prolonged length of stay in the hospital as opposed to their non-obese counterparts. ${ }^{13}$ Moreover, the authors found that there was a $12 \%$ increment in the risk of 
developing severe COVID-19 with every one unit escalation in the body mass index (BMI). Another study, also from China, clearly elucidated that a higher BMI is associated with more severe disease manifestations. ${ }^{14}$

NAFLD (the presence of fat in the liver, after excluding other causes like significant alcohol consumption or certain drugs) is a clinical spectrum which ranges from liver steatosis to advanced fibrosis, cirrhosis and even hepatocellular carcinoma (HCC). ${ }^{15}$ However, patients with NAFLD often have other metabolic derangements like presence of type 2 Diabetes mellitus, hypertension, dyslipidemia and obesity. ${ }^{14}$ It was also found that this subset of patients progressed rapidly to liver cirrhosis and its complications. Therefore, the term MAFLD was introduced as a practical terminology to identify such patients and to minimise their risk factors more aggressively in order to prevent liver decompensation. ${ }^{16}$

There are a fair amount of upcoming data regarding the potential association of MAFLD with COVID-19 disease severity. One retrospective analysis presented data of 202 confirmed cases of COVID-19 (diagnosed by SARS-COV-2 PCR in throat swab). ${ }^{17}$ This study showed that although liver injury in majority of the COVID positive patients was of a relatively milder nature, patients with concomitant MAFLD had a higher probability of liver dysfunction and increased risk of disease progression. Moreover, the same study revealed that these patients shed their virus for a prolonged period of time as compared to individuals without underlying MAFLD. Another study on 310 subjects (approximately one-third of which were MAFLD patients) looked at the association between the degree of fibrosis (based fibrosis-4 \{FIB-4)\} index and NAFLD fibrosis score) and severity of COVID infection. Here also, the results showed that the severity of illness markedly increased (in terms of high CRP, lymphopenia and thrombocytopenia) in MAFLD patients with moderate or high fibrosis scores. ${ }^{18}$ The authors hypothesised that MAFLD with significant liver fibrosis could potentially aggravate the cytokine storm triggered by the viable virus, which may be responsible forillness severity.

The impact of age on the severity of COVID-19 patients with MAFLD was analysed by a multicentre study, which broadly divided patients into two groups: younger ( $<60$ years) and elderly (>60 years). Interestingly, the authors concluded that younger individuals had a positive correlation between MAFLD and the severity of COVID-19; whereas, no such association was found among older patients with NAFLD. ${ }^{19}$ Again, the postulated mechanism explaining this phenomenon may be the hepatic immune responses (involving the activation of dendritic cells, T-lymphocytes, natural killer cells and macrophages), resulting in raised cytokine levels seen in patients with MAFLD. ${ }^{20}$ These immune responses are more robust in younger subjects; hence, the likelihood of such patients going into cytokine storm is higher as against to older people. However, due to small sample size of this study, the validity of results may not be truly established.

There are a lot of grey areas in research as far as MAFLD and
COVID-19 are concerned. Firstly, validity of most studies may not be truly established as most published studies have got a modest sample size and; hence, lesser generalisability of results. Secondly, it is so far not very evident whether MAFLD, with or without obesity, is alone responsible for the catastrophic outcome in patients with COVID-19. These individuals may also have coexisting chronic illnesses like type 2 Diabetes mellitus, hypertension and hyperlipidemias, which can further aggravate the viral illness. ${ }^{21}$ Finally, there is a deficiency of information regarding previous history of liver disease in COVID-19 patients as a whole. ${ }^{22}$ This becomes even more intricate for patients with MAFLD as most of them remain undetected in the initial phase, when the disease is asymptomatic.

COVID-19 has posed an immense burden on the entire world, both in terms of the disease and its complications, as well as the exorbitant financial implications associated with it. Internationally, many healthcare systems are on the verge of collapse due to the universal pandemic. Considering the fact that global prevalence of obesity and MAFLD is also on the rise, a significant proportion of the world population could be in danger of developing grave complications of COVID-19. At this point of time, we may merely be witnessing the tip of the iceberg. Therefore, it is highly imperative that further high quality research with larger sample size be conducted in order to fully comprehend the disease pathophysiology; and eventually work towards effective disease control and prevention.

\section{REFERENCES}

1. Zhu N, Zhang D, Wang W. A novel coronavirus from patients with pneumonia in China, 2019. N Engl J Med 2020; 382(8): 727-33. doi:10.1056/NEJMoa2001017.

2. Peiris JS, Yuen KY, Osterhaus AD, Stöhr K. The severe acute respiratory syndrome. N Engl J Med 2003; 349(25): 2431441. doi:10.1056/NEJMra032498.

3. Guan WJ, Ni ZY, Hu Y. Clinical characteristics of coronavirus disease 2019 in China.N Engl J Med 2020; 382(18): 1708-20. doi:10.1056/NEJMoa2002032.

4. Ridruejo E, Soza A. The liver in times of COVID-19: What hepatologists should know. Ann Hepatol 2020; 19(4):353-8. doi:10.1016/j.aohep.2020.05.001.

5. Younossi ZM, Henry L. Economic and quality-of-life implications of non-alcoholic fatty liver disease. Pharmacoeconomics 2015; 33(12):1245-53. doi:10.1007/s40273-0150316-5.

6. Eslam M, Newsome PN, Sarin SK. A new definition for metabolic dysfunction-associated fatty liver disease: An international expert consensus statement. J Hepatol 2020; 73(1):202-9. doi:10.1016/j.jhep.2020.03.039.

7. Zhang C, Shi L, Wang FS. Liver injury in COVID-19: Management and challenges. Lancet Gastroenterol Hepatol 2020; 5(5):428-30. doi:10.1016/S2468-1253(20)30057-1.

8. Xu Z, Shi L, Wang Y. Pathological findings of COVID-19 associated with acute respiratory distress syndrome [published correction appears in Lancet Respir Med. 2020 Feb 25]. Lancet Respir Med 2020; 8(4):420-2. doi:10.1016/ S2213-2600(20)30076-X.

9. Chai X, Hu L, Zhang Y, Han W, Lu Z, Ke A, et al. Specific 
ACE2 expression in cholangiocytes may cause liver damage after 2019-nCoV infection. bioRxiv 2020. doi: http:// dx.doi.org/10.1101/2020.02.03.931766.

10. Gacouin A, Locufier M, Uhel F. Liver cirrhosis is independently associated with 90-day mortality in ARDS patients. Shock 2016; 45(1):16-21. doi:10.1097/SHK. 0000000000000487.

11. Monteiro CA, Moura EC, Conde WL, Popkin BM. Socioeconomic status and obesity in adult populations of developing countries: A review. Bull World Health Organ 2004; 82(12):940-946.

12. Murugan AT, Sharma G. Obesity and respiratory diseases. Chron Respir Dis 2008; 5(4):233-42. doi:10.1177/ 1479972308096978.

13. Gao $\mathrm{F}$, Zheng $\mathrm{Kl}$, Wang $\mathrm{XB}$. Obesity is a risk factor for greater COVID-19 severity. Diabetes care 2020; 43(7):e72-e74. doi:10.2337/dc20-0682.

14. Cai Q, Huang D, Ou P. COVID-19 in a designated infectious diseases hospital outside Hubei Province, China. Allergy 2020; 75(7):1742-52. doi:10.1111/all.14309.

15. Leoni S, Tovoli F, Napoli L, Serio I, Ferri S, Bolondi L. Current guidelines for the management of non-alcoholic fatty liver disease: A systematic review with comparative analysis. World J Gastroenterol 2018; 24(30):3361-73. doi:10.3748/wjg.v24.i30.3361.

16. Lin S, Huang J, Wang M. Comparison of MAFLD and NAFLD diagnostic criteria in real world [published online ahead of print, 2020 Jun 1]. Liver Int 2020; 10.1111/liv.14548. doi:10.1111/liv.14548.

17. Ji D, Qin E, Xu J. Non-alcoholic fatty liver diseases in patients with COVID-19: A retrospective study [published online ahead of print, 2020 Apr 8]. J Hepatol 2020; S01688278(20):30206-3. doi:10.1016/j.jhep.2020.03.044.

18. Targher G, Mantovani A, Byrne CD. Risk of severe illness from COVID-19 in patients with metabolic dysfunctionassociated fatty liver disease and increased fibrosis scores. Gut 2020; 69(8):1545-1547. doi:10.1136/ gutjnl2020-321611.

19. Zhou YJ, Zheng KI, Wang XB. Younger patients with MAFLD are at increased risk of severe COVID-19 illness: A multicenter preliminary analysis [published online ahead of print, 2020 Apr 26]. J Hepatol 2020; S0168- 8278 (20):30271-3. doi:10.1016/j.jhep.2020.04.027.

20. Narayanan S, Surette FA, Hahn YS. The immune landscape in nonalcoholic steatohepatitis. Immune Netw 2016; 16(3):147-58. doi:10.4110/in.2016.16.3.147.

21. Boettler T, Newsome PN, Mondelli MU. Care of patients with liver disease during the COVID-19 pandemic: EASL-ESCMID position paper. JHEP Rep 2020; 2(3):100113. doi:10.1016/ j.jhepr.2020.100113.

22. Mantovani A, Beatrice G, Dalbeni A. Coronavirus disease 2019 and prevalence of chronic liver disease: A metaanalysis. Liver Int 2020; 40(6):1316-20. doi:10.1111/ liv. 14465. 